\title{
Is the "sterile cockpit" concept applicable to cardiovascular surgery critical intervals or critical events? The impact of protocol-driven communication during cardiopulmonary bypass
}

\author{
Rishi K. Wadhera, BS, ${ }^{a}$ Sarah Henrickson Parker, MS, ${ }^{\mathrm{b}}$ Harold M. Burkhart, MD, ${ }^{\mathrm{a}}$ Kevin L. Greason, MD, ${ }^{\mathrm{a}}$ \\ James R. Neal, CCP, ${ }^{\mathrm{a}}$ Katherine M. Levenick, CCP, ${ }^{\mathrm{a}}$ Douglas A. Wiegmann, $\mathrm{PhD},{ }^{\mathrm{c}}$ and Thoralf M. Sundt III, $\mathrm{MD}^{\mathrm{a}}$
}

\begin{abstract}
Objective: There is general enthusiasm for applying strategies from aviation directly to medical care; the application of the "sterile cockpit" rule to surgery has accordingly been suggested. An implicit prerequisite to the evidence-based transfer of such a concept to the clinical domain, however, is definition of periods of high mental workload analogous to takeoff and landing. We measured cognitive demands among operating room staff, mapped critical events, and evaluated protocol-driven communication.
\end{abstract}

\begin{abstract}
Methods: With the National Aeronautics and Space Administration Task Load Index and semistructured focus groups, we identified common critical stages of cardiac surgical cases. Intraoperative communication was assessed before $(n=18)$ and after $(n=16)$ introduction of a structured communication protocol.
\end{abstract}

Results: Cognitive workload measures demonstrated high temporal diversity among caregivers in various roles. Eight critical events during cardiopulmonary bypass were then defined. A structured, unambiguous verbal communication protocol for these events was then implemented. Observations of 18 cases before implementation including 29.6 hours of cardiopulmonary bypass with 632 total communication exchanges (average 35.1 exchanges/case) were compared with observations of 16 cases after implementation including 23.9 hours of cardiopulmonary bypass with 748 exchanges (average 46.8 exchanges/case, $P=.06$ ). Frequency of communication breakdowns per case decreased significantly after implementation (11.5 vs 7.3 breakdowns/case, $P=.008$ ).

Conclusions: Because of wide variations is cognitive workload among caregivers, effective communication can be structured around critical events rather than defined intervals analogous to the sterile cockpit, with reduction in communication breakdowns. (J Thorac Cardiovasc Surg 2010;139:312-9)

Effective communication is a critical precondition of effective teamwork and high-reliability performance in highrisk and high-consequence environments such as the cardiac surgical operating room (OR). It is therefore not surprising that breakdowns in communication in the OR have been linked to worse surgical outcomes. ${ }^{1,2}$ In an interview study of consultant surgeons, incomplete, nonexistent, or erroneous communication was a causal factor in $43 \%$ of surgical adverse events, ${ }^{3}$ and a recent surgeon review of 444 surgical malpractice claims identified 81 communication breakdowns among 60 of these claims, $92 \%$ of which were verbal interchanges or events. ${ }^{4}$

Unfortunately, ineffective communication is not uncommon in the OR. In an observational study of general and vascular surgical ORs, Lingard and colleagues ${ }^{5}$ documented

\footnotetext{
From the Mayo Clinic, ${ }^{\mathrm{a}}$ Rochester, Minn; the University of Aberdeen, ${ }^{\mathrm{b}}$ Aberdeen, Scotland; and the University of Wisconsin-Madison, ${ }^{\mathrm{c}}$ Madison, Wis. Disclosures: None.

Read at the Thirty-fifth Annual Meeting of The Western Thoracic Surgical Association, Banff Springs, Alberta, Canada, June 24-27, 2009.

Received for publication June 29, 2009; revisions received Oct 4, 2009; accepted for publication Oct 25, 2009.

Address for reprints: Thoralf M. Sundt III, MD, Division of Cardiovascular Surgery, Mayo Clinic, 200 First St SW, Rochester, MN 55905 (E-mail: sundt.thoralf@mayo.edu). 0022-5223/\$36.00

Copyright (c) 2010 by The American Association for Thoracic Surgery doi: $10.1016 /$ j.jtcvs. 2009.10 .048
}

"communication failures," as defined by poor timing, inaccurate or incomplete information, failure to include key team members or failure to resolve issues, in $31 \%$ of OR communications. A third of these failures resulted in demonstrable inefficiency, increased cognitive workload, interruption of flow, and increased tension, as well as wasted resources. Despite their negative impact on the work environment, such failures may remain unresolved, because staff members often use process work-arounds that may solve immediate challenges but do not address long-term systemic inadequacies. ${ }^{6}$ Specifically, within the domain of cardiovascular surgery, our group has previously shown that communication failures adversely affect technical surgical performance. ${ }^{7}$ Unfortunately, consistent with the observations of others, we have also found that only a third of nonphysician caregivers in our ORs consider surgeon communication to be effective. ${ }^{8,9}$

The cardiovascular surgical OR is clearly a high-consequence environment; it is also a high-risk environment. The cardiovascular surgical OR is characterized by a high degree of complexity with respect to both human-technology interfaces and human-human interfaces. In addition to sophisticated technologies such as cardiopulmonary bypass (CPB) machines, individuals filling as many as 9 different roles (surgeon, resident, perfusionist, anesthesiologist, 


\section{Abbreviations and Acronyms \\ $\mathrm{ACT}=$ activated clotting time \\ $\mathrm{CPB}=$ cardiopulmonary bypass \\ NASA TLX $=$ National Aeronautics and Space Administration Task Load Index \\ OR = operating room}

Aeronautics and Space Administration Task Load Index (NASA TLX) a validated tool to measure mental workload. ${ }^{14}$ The NASA TLX was administered to 30 cardiovascular OR staff, including perfusionists, certified registered nurse anesthetists, surgical assistants, surgical technicians, and circulating nurses. One NASA TLX was filled out for each of 8 different stages of surgery (preparation, induction, opening, initiation of CPB, surgical repair, termination of $\mathrm{CPB}$, closure, and postoperative), giving a stageby-stage assessment of mental workload. Scores were assessed separately for representatives of each role.

\section{Assessment of Critical Stages}

Critical stages of a typical cardiac surgical case were used to identify critical stages of a typical cardiovascular surgical case in general and of a typical $\mathrm{CPB}$ run in particular through semistructured focus groups with surgeons and perfusionists. For the CPB interval, stages were assessed for critical communication required and informally assessed for potential risk should communication break down. Eight critical events common to most CPB runs were determined. Standard phraseology for each critical stage was also explored through interview with experienced perfusionists, who were asked to determine the desired general structure and content for each stage. After several iterations, a final communication protocol was developed. During the implementation period, the protocol was posted on the wall opposite the consultant surgeon in the OR in large font on 2 laminated sheets of $8.5 \times 11$-inch paper. The perfusionist was also provided with a copy to keep at the heart-lung machine. This was done to ensure that implementation of the protocol did not further increase mental workload by forcing the surgical team members to remember a "script."

dressed similar challenges by instituting protocol-driven communication and a "sterile cockpit" rule during periods of high risk and high mental workload. These periods include taxi, takeoff, and landing. During these events, nonessential activities are prohibited, and discussion in the cockpit must be exclusively related to handling the aircraft. Once cruising altitude has been achieved, the risk decreases and the pilots may discuss whatever they please. ${ }^{12}$ Standardized communication protocols or standard phraseology were developed to decrease ambiguity and expedite the communication process between pilots and air traffic control, ${ }^{13}$ as was uniform application of repetition or "call-back" of critical information. $^{12}$

There is general enthusiasm for applying strategies from aviation directly to medical care, and the application of the sterile cockpit concept to the OR has been suggested. An implicit prerequisite for the evidence-based transfer of such a concept to the clinical domain, however, is the clear definition of periods of high mental workload. As a first step, we sought to determine the applicability of the same principles to the cardiovascular surgical OR by mapping critical events as well as cognitive demands among all members of the OR team throughout a surgical procedure. On the basis of these findings, we further developed and implemented a protocolbased communication tool specifically for the surgeon-perfusionist axis and assessed its impact on the effectiveness of communication.

\section{MATERIALS AND METHODS}

\section{Assessment of Mental Workload}

We assed the level of cognitive workload during an entire cardiac surgical procedure from the perspective of all involved staff with the National

\section{Collection and Analysis of Communication Exchanges}

Observations were made at St Marys Hospital at Mayo Clinic, Rochester, Minn, with the consent of all surgeons and staff members. Institutional review board approval was granted for a minimal risk study with study-specific consent by patients waived. Observations were made by 1 of 2 independent observers (R.K.W., S.H.P.), with details of exchanges recorded manually. Before implementation of the protocol, communication exchanges between cardiac surgeon and perfusionist were recorded verbatim in 18 cardiovascular surgical cases during CPB to assess the forms and frequency of miscommunication. The protocol was then implemented in 16 cardiovascular surgical cases, and communication exchanges between car$\mathrm{CPB}$. Cases observed were a convenience sample from among the elective operative schedule of 6 cardiovascular surgeons.

Communication exchanges between cardiac surgeon and perfusionist before and after implementation of the standard communication protocol were categorized as either no issue or as one of the following types of communication breakdowns: (1) miscues, (2) no call-back (either surgeon or perfusionist), (3) repeated communication exchanges, (4) occurrence of a nonverbalized critical action, and (5) ambiguous or unstructured communication exchanges. These categorizations were determined before observations on the basis of our previous work in the cardiac surgical OR. Definitions of categories are shown in Table 1. Categorization of each communication event as no issue, miscue, no call-back, repeated communication event, occurrence of nonverbalized critical action, or ambiguous or unstructured communication event was validated by independent categorization by 2 raters (R.K.W., S.H.P.), and agreement was determined. Observed communication exchange data were also used to determine the frequency at which no communication or poor communication occurred during each critical stage.

\section{Statistical Analysis}

The number of cases observed was based on predetermined approximations of sample sizes that would give $80 \%$ power at a .05 level of diac surgeon and perfusionist were again monitored and recorded during 
TABLE 1. Communication breakdown definitions

\begin{tabular}{ll}
\hline \multicolumn{1}{c}{ Type of breakdown } & Definition \\
\hline No call-back & Exchanges between surgeon and perfusionist in which confirmatory call-back of action or \\
& instruction did not occur \\
Repeated communication exchange & A communication exchange that must be repeated \\
Nonverbalized critical action & Occurrence of actions that were not communicated between surgeon and perfusionist \\
Ambiguous or unstructured communication exchange & Communication exchanges that do not include specific instructions, (eg, cardioplegia given \\
& antegrade or retrograde) \\
Miscues & Surgeon or perfusionist not clearly hearing communication exchanges or requests from one \\
& another, either because of volume or because of confusion \\
\hline
\end{tabular}

significance (2-sided). Descriptive analyses were used to assess the frequencies of types of communication breakdown between cardiac surgeon and perfusionist before and after implementation of the standardized communication protocol. A rank sum Wilcox test was used to compare mean number of communication breakdowns.

\section{RESULTS \\ Mental Workload}

The results of the NASA TLX showed widely divergent cognitive workload measures during the course of a typical case, as shown in Figure 1. For example, during induction and intubation, workload is high for anesthesia but not for other disciplines. Although this is clearly a high-risk interval, commonly individuals in other disciplines will be focusing on their own work during this period, often chatting, preparing or counting instruments on the sterile table, or so on, which may challenge the mental focus of the anesthesia staff. These results suggest that, in contrast to the circumstance in aviation, there is no discrete time period that may be conveniently defined as the principal period of highrisk and high mental workload from the standpoint of the entire team.

\section{Mental Workload in the Operating Room NASA-Task Load Index (NASA TLX) $(n=30)$}

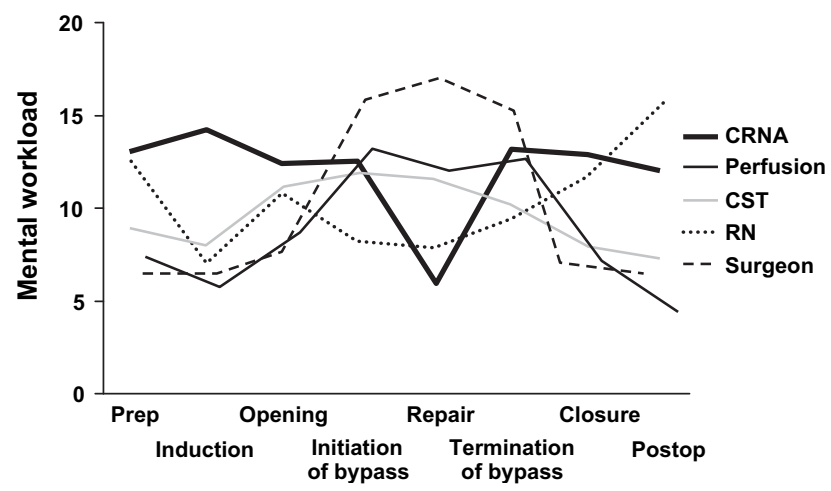

FIGURE 1. Results of National Aeronautics and Space Administration Task Load Index (NASA TLX) show widely divergent cognitive workload measures during course of typical case. CRNA, certified registered nurse anesthetist; CST, certified surgical technologist; $R N$, registered nurse; Prep, surgical preparation; Postop, postoperative.

\section{Identification of Critical Stages and Communication Protocols}

With the focus group methodology, consensus was reached among staff (cardiac surgeons and perfusionists) regarding the critical common events during $\mathrm{CPB}$ as shown in Table 2, including (1) establishment of activated clotting time (ACT), (2) circuit check, (3) initiation of CPB, (4) crossclamp on, (5) administration of cardioplegia, (6) vent on and off, (7) crossclamp off, and (8) termination of CPB. It is important to note that although these critical stages are evident across most case types, some cases may not require the use of every single stage.

After definition of the 8 critical events, a communication protocol was developed that was directed toward delineation of the vital information that needed to be exchanged at each stage and establishment of a structured means to convey this information. Specifically, the format of the exchange included specific information pertaining to the case, rather than vague indicators. For example, for the critical step of "ACT adequate for CPB," the protocol called for the numeric ACT level to be given, rather than merely stating " adequate for CPB." Furthermore, on the basis of the principles noted previously, call-back of all exchanges was instituted as a standard procedure. There was considerable debate regarding whether specific communication protocols should be developed for each procedure type; however, it was determined that for the sake of enhanced utility and adoption a single universal communication protocol should be used.

\section{Communication Breakdowns}

Eighteen cardiovascular surgical cases were observed before implementation of the communication protocol and 16

TABLE 2. Critical stages of cardiopulmonary bypass

Activated clotting time adequate, arterial line check
Circuit check
Initiation of cardiopulmonary bypass
Crossclamp on
Cardioplegia updates
Vent updates
Crossclamp off
Termination of cardiopulmonary bypass


TABLE 3. Classification of observed procedure types

\begin{tabular}{lcc}
\hline \multicolumn{1}{c}{ Procedure } & $\begin{array}{c}\text { Preimplementation } \\
\text { cases }\end{array}$ & $\begin{array}{c}\text { Postimplementation } \\
\text { cases }\end{array}$ \\
\hline CABG & 5 & 4 \\
CABG with valve repair & 2 & 2 \\
Valve repair or replacement & 6 & 7 \\
Descending or ascending & 3 & 0 \\
$\quad$ aorta repair & & 3 \\
Other & 2 & \\
$C A B G$, Coronary artery bypass grafting. &
\end{tabular}

after implementation, as shown in Table 4. Procedures were performed by 6 different surgeons. Before implementation, communication exchanges between cardiac surgeon and perfusionist were observed and recorded for a total of 29.6 hours (1775 minutes) of CBP time. After implementation, communication exchanges were observed for a total of 23.9 hours (1433 minutes). The average durations of CPB before and after implementation (98.6 vs 89.5 minutes, $P=.55$ ) and cases mixes were similar (Table 3 ).

With regard specifically to the 8 critical stages, breakdowns before implementation most frequently concerned application ( $65 \%$ of cases) or removal (65\% of cases) of the aortic crossclamp (Figure 2). Of note, the breakdowns related to application of the crossclamp were entirely due to lack of verbalization, whereas breakdowns on removal were not verbalized in $47 \%$ of cases, were ambiguous in $12 \%$, and were without call-back or acknowledgment in $6 \%$. In addition, in $22 \%$ of cases there was no verbalized confirmation of an adequately functioning circuit (satisfactory arterial line pressure oxygenation, etc). Perhaps of greater concern, there was no verbalized assurance regarding an adequate $\mathrm{ACT}$ for $\mathrm{CPB}$ in $11 \%$ of cases.

After implementation of the communication protocol, the frequency of ineffective communication of the critical events improved. Specifically, the frequency of a missed verbalized circuit check was $6 \%$ of cases, which marked a significant decrease from $22 \%$ of cases before implementation. No or poor verbalization concerning the aortic crossclamp remained rather common at $33 \%$ after use of the protocol, although this was much improved from the level before the institution of the protocol. Furthermore, verbal- ization that the crossclamp had been removed occurred in all cases after implementation, in contrast lack of verbalization in $65 \%$ of case before implementation.

Moreover, communication improved broadly after implementation of the protocol. Overall, during the 18 preimplementation cases, 632 total communication exchanges occurred between cardiac surgeon and perfusionist. During the 16 postimplementation cases, 748 total communication exchanges were observed. The average numbers of communication exchanges per case were similar between preimplementation and postimplementation groups (35.1 vs 46.8 , $P=.06)$. The total frequency of communication breakdowns per case decreased significantly after implementation of the communication protocol (11.5 vs 7.3, $P=.008)$.

Examples of a nonverbalized critical action included the perfusionist turning on the left ventricular vent when the surgeon wanted it off, the surgeon removing the crossclamp without informing the perfusionists, or initiation of rewarming without a shared understanding. After the introduction of the communication protocol, nonverbalized critical actions per case were reduced by approximately $75 \%$ (1.6 vs 0.4 , $P=.004)$. In addition, the number of communication exchanges that required repetition per case decreased appreciably with the communication protocol (1.4 vs $0.3, P=.042$ ).

Call-backs are often used between surgeon and perfusionist to verify communication exchanges within this communication axis. The frequency per case at which call-backs did not occur between surgeon and perfusionist decreased $26 \%$ in the postimplementation group, but this difference was not statistically significant (3.9 vs $2.9, P=.20$ ). Miscues were defined as surgeon or perfusionist incorrectly hearing or interpreting a communication exchange or request. There was no appreciable difference in the frequency of miscues between preimplementation and postimplementation groups (1.2 vs 1.4, $P=.57$ ). Examples of ambiguous or unstructured communication exchanges included the surgeon requesting cardioplegia without specifying dose or antegrade versus retrograde and vague or nonspecific communication exchanges (such as "off," "up," or "down") that elicited a confused response from the perfusionist. Ambiguous or unstructured communication exchanges per case decreased $32 \%$ after implementation of

TABLE 4. Changes in communication breakdowns

\begin{tabular}{|c|c|c|c|c|}
\hline Variable & Preimplementation & Postimplementation & Change & $P$ value \\
\hline $\begin{array}{l}\text { Total communication } \\
\text { breakdowns }\end{array}$ & 11.5 & 7.3 & $-37 \%$ & .008 \\
\hline Nonverbalized critical actions & 1.6 & 0.4 & $-75 \%$ & .004 \\
\hline Repeated communication & 1.4 & 0.3 & $-79 \%$ & .042 \\
\hline No call-back & 3.9 & 2.9 & $-26 \%$ & .20 \\
\hline Miscues & 1.2 & 1.4 & $16 \%$ & .57 \\
\hline $\begin{array}{l}\text { Ambiguous or unstructured } \\
\text { communication exchanges }\end{array}$ & 3.4 & 2.3 & $-32 \%$ & .67 \\
\hline
\end{tabular}

Preimplementation and postimplementation values represent breakdowns per case. 


\section{Proportion of Cases with Communication Breakdowns at Each of 8 "Critical Events"}

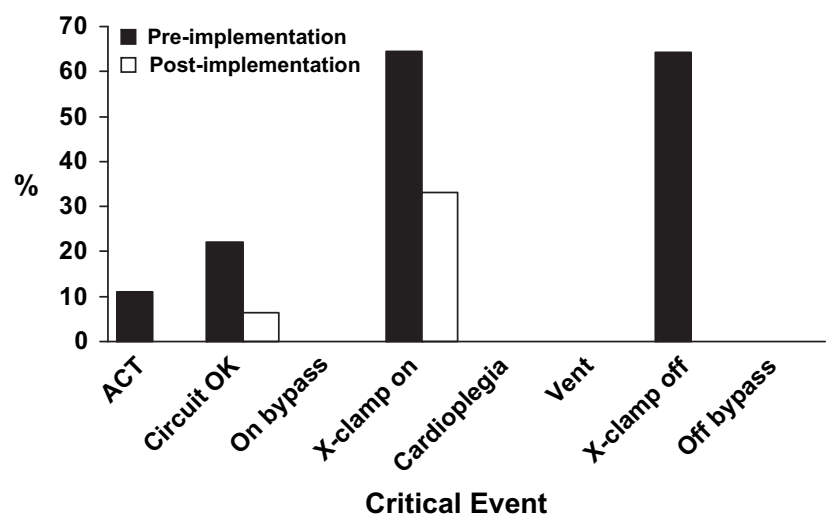

FIGURE 2. Proportions of cases with communication breakdowns at each of 8 critical events defined in study. ACT, Activated clotting time; $X$-clamp, aortic crossclamp.

the communication protocol, but this change did not attain statistical significance (3.4 vs $2.3, P=.67$ ).

\section{DISCUSSION}

The results of this study demonstrate that, in contrast to aviation, there is no discrete time interval of high risk and high mental workload in cardiac surgery that can be defined during which a sterile cockpit rule can be readily implemented. Because of the overall complexity of a surgical procedure and the diversity of tasks to be accomplished by individuals with different roles throughout the procedure, an alternative approach focusing on "critical events" rather than a "critical time interval" may be more useful. Furthermore, our study suggests that a structured focus on communication around such events can have a positive effect beyond the events themselves, with an improvement in the quality of communication in general. Although the intervention tested here focused only on the surgeon-perfusionist axis, the tool could likely be expanded to include the other critical events in the OR, including intubation, surgical pause or incision, instrument and sponge counts, and so on.

We have previously demonstrated that disruptions in the flow of the operation, particularly those related to communication failure, correlate with technical error. ${ }^{7}$ We have also demonstrated that a preoperative briefing can reduce miscommunication events by half even when introduced with a highly familiar team. ${ }^{15}$ This study builds on these findings while focusing specifically on the surgeon-perfusionist axis. We have demonstrated a significant opportunity to improve performance, with almost a third of exchanges between surgeon and perfusionist judged suboptimal. Furthermore, a simple, low-cost intervention that did not depend on expensive technology was able to affect the measured outcome, reducing miscommunication by half. The communication protocol was deliberately generic, designed for applicability across a broad range of cases.

The communication protocol had its greatest effect by diminishing nonverbalized critical actions and repeated communication exchanges, suggesting that the protocol served as an aid particularly during stages of high mental workload. In particular, application and removal of the crossclamp occur during a period of high mental workload for both the surgeon and perfusionist and are accordingly at high risk for loss of situational awareness as a result of task focus. The protocol established a reminder to the surgeon to be aware of the perfusionist's "need to know" and also set an expectation by the perfusionist for receipt of a communication event. The latter may account for the improvement in repeated exchanges. Both the reduction in ambiguous communication exchanges, as defined by absence of quantitatively specific instructions, and the improvement in adherence to call-back during communication exchanges were modest. Call-backs are particularly problematic behaviorally, because they tend to benefit the initiator of the communication, allowing verification that the receiver has heard the communication exchange. Therefore the onus is on the receiver to respond, yet a response may not seem necessary if the recipient feels that he or she has adequately heard and understood the communication. The occurrence of miscues actually appeared to increase modestly; however, this difference was not statistically significant.

Quantification of the potential impact of this intervention on adverse events is difficult; however, it is well documented that failures in communication are frequent contributors to adverse events. ${ }^{1-3}$ It is therefore reasonable to anticipate that improvement in communication generally will reduce the risk of error and, when an error occurs, improve the ability of the team to identify and recover from it. As stated by Salas and colleagues, ${ }^{16}$ communication is a fundamental element of effective teamwork, along with coordination and cooperation. Accordingly, express attention to communication through the introduction of protocol-based communication can reasonably be expected to promote active engagement of all team members, with the resultant benefits of mutual performance monitoring, mutual support, team orientation, and cohesion. Our previous work has demonstrated that the state of teamwork in general, and communication specifically, is not perceived positively by all members of the OR team, and in fact are thought to be poor by as many as half of the nonphysician staff members. ${ }^{8}$ Our admittedly anecdotal experience during this study was that the OR team was more engaged and willing to speak up with concerns for patient safety when the importance of effective communication was acknowledged and valued.

Research in other high consequence industries has demonstrated several critical characteristics of high reliability organizations. ${ }^{17}$ These organizations are characterized by the mindful management of operations through (1) 
preoccupation with the possibility of and modes of failure, (2) reluctance to simplify interactions, (3) sensitivity to operations, (4) a commitment to resilience, and (5) deference to operational expertise. Effective communication is an essential precondition to all of these factors, and in our opinion communication improvement demands a conscious and intentional approach with consideration of lessons learned in such other high consequence industries as nuclear power, aviation, and the military.

There are significant limitations to this study. The study was performed in a single institution and with a small number of surgeons who were inclined to accept the value of improved communication among team members. We were, however, able to include a diversity of different surgeonperfusionist communication dyads, as well as a large number of case types. A second limitation was the availability of only 2 human factor observers, with no technical capability to continuously record the actual exchanges in such a manner that they could be "over read" or reviewed by other investigators. Both observers kept word-for-word records of the communications between the surgeon and the perfusionist, however, leaving the judgment of what did and did not constitute a communication breakdown for later coding analysis and discussion. Coding was performed independently, with results compared only after all the data had been analyzed. Finally, there is no means of accounting for the potential for the Hawthorne effect of improved performance simply because observers are present. This effect, however, would have been expected to improve communication both before and after institution of the protocol and should not, therefore, invalidate our positive results.

More broadly, the results are limited by the interval of observation and the difficulty in assessing adherence to the protocol with time and its lasting impact on behavior in the OR. True change in the culture of a complex environment is gradual, and there is a tremendous pull toward return to the stable state that existed before the intervention. Effective surgical leadership is critical if such changes are to be incorporated into the fabric of the environment. Perhaps the most important element is demonstration to surgical trainees of the importance and impact of effective communication exchange as a critical element of teamwork.

In summary, the results of this study demonstrate that in light of the complexity of cardiovascular care in the OR, a sterile cockpit approach based on the definition of a critical time interval is not as applicable as is an approach focused on critical events. Furthermore, the institution of such a protocol had a positive effect on communication throughout the procedure. Similar protocols should be readily customizable to most practice environments, with no significant disruption to the flow of the procedure itself because they do not constitute a pause or checklist, and such interventions have the potential to significantly improve outcomes.
We gratefully acknowledge the assistance of Jeffrey Riley, CCP, in the phase of data collection, as well as the generous and patient support of the entire OR staff during conduct of this study.

\section{References}

1. Carthey J, de Leval MR, Reason JT. The human factor in cardiac surgery: errors and near misses in a high technology medical domain. Ann Thorac Surg. 2001;72: $300-5$.

2. de Leval MR, Carthey J, Wright DJ, Farewell VT, Reason JT. Human factors and cardiac surgery: a multicenter study. J Thorac Cardiovasc Surg. 2000;119: 661-72.

3. Gawande AA, Zinner MJ, Studdert DM, Brennan TA. Analysis of errors reported by surgeons at three teaching hospitals. Surgery. 2003;133:614-21.

4. Greenberg CC, Regenbogen SE, Studdert DM, Lipsitz SR, Rogers SO, Zinner MJ, et al. Patterns of communication breakdowns resulting in injury to surgical patients. J Am Coll Surg. 2007;204:533-40.

5. Lingard L, Espin S, Whyte S, Regehr G, Baker GR, Reznick R, et al. Communication failures in the operating room: an observational classification of recurrent types and effects. Qual Saf Health Care. 2004;13:330-4.

6. Tucker AL, Edmonson AC. Why hospitals don't learn from failures: organizational and psychological dynamics that inhibit system change. Calif Manage Rev. 2003;45:55-72.

7. Wiegmann DA, ElBardissi AW, Dearani JA, Daly RC, Sundt TM 3rd. Disruptions in surgical flow and their relationship to surgical errors: an exploratory investigation. Surgery 2007; 1 142:658-665.

8. ElBardissi AW, Wiegmann DA, Dearani JA, Daly RC, Sundt TM III. Application of the human factors analysis and classification system methodology to the cardiovascular surgery operating room. Ann Thorac Surg. 2009;83:1412-5.

9. Flin R, Yule S, McKenzie L, Paterson-Brown S, Maran N. Attitudes to teamwork and safety in the operating theatre. Surgeon. 2006;4:145-51.

10. Wiegmann DA, Suther T, Neal J, Parker SH, Sundt TM. A human factors analysis of cardiopulmonary bypass machines. J Extra Corpor Tech. 2009;41:57-63.

11. ElBardissi AW, Wiegmann DA, Dearani JA, Daly RC, Sundt TM 3rd. Application of the human factors analysis and classification system methodology to the cardiovascular surgery operating room. Ann Thorac Surg. 2007;83:1412-9.

12. Sumwalt RL. The sterile cockpit. ASRS Directline [Internet] 1993 Jun [cited 2009 Dec 19];4:[about 1 p.] Available from: http://asrs.arc.nasa.gov/publications/ directline/dl4_sterile.htm

13. ICAO Standard Phraseology Manual. [cited 2009 Dec 18] Available from: http:// www.skybrary.aero/bookshelf.books/115.pdf

14. Hart SG, Staveland LE. Development of NASA-TLX (task load index)a multi-dimensional workload rating scale: results of empirical and theoretical research. In Hancock PA, Meshkati N, eds. Advances in psychology 52: human mental workload. Amsterdam: Elsevier Science; 1988:139-83.

15. Henrickson SE, Wadhera RK, Elbardissi AW, Wiegmann DA, Sundt TM 3rd. Development and pilot evaluation of a preoperative briefing protocol for cardiovascular surgery. J Am Coll Surg. 2009;208:1115-23.

16. Salas E, Wilson KA, Murphy CE, King H, Salisbury M. Communicating, coordinating, and cooperating when lives depend on it: tips for teamwork. Jt Comm J Qual Patient Saf. 2008;34:333-41.

17. Weick KE, Sutcliffe KM. Managing the unexpected: resilient performance in an age of uncertainty. Hoboken (NJ): Wiley; 2001.

\section{Discussion}

Dr James I. Fann (Palo Alto, Calif). Dr Sundt, thank you for a comprehensive and compelling presentation on this patient safety issue. The concept of sterile cockpit is well established in the field of aviation, with Federal Aviation Administration regulations specifically mandating that pilots refrain from nonessential activities during critical phases of flight. In the context of surgery, on first pass, one might consider the sterile cockpit model to be applicable because of the common perception that the surgeon has a role analogous to that of the pilot. But is this aviation model of the sterile cockpit directly applicable to the cardiac surgical environment? 
That question, along with a potential solution in a communication protocol, is thoughtfully analyzed in this study.

Errors in surgery and in medicine in general often can be traced to errors in communication, as was mentioned, but such generalities regarding the need for more effective communication have not previously translated into a well-designed study or a practical protocol for improvement in the field of cardiothoracic surgery. The surgical checklist, for instance, which is also based on the aviation model, focuses on preoperative and postoperative debriefing and has been shown to reduce errors and improve patient outcomes. Further improvement in surgical outcomes will require us to recognize other complex intraoperative components that make up the cardiac surgical environment.

The importance of this study is twofold. It graphically demonstrates the divergence and complexity of tasks among the diverse team members. In effect, in the cardiac surgery environment, in terms of the cognitive workload, instead of having a single cockpit as is the case with aviation has multiple cockpits, with implications for patient safety. It is well recognized that there are 3 tribes in the surgical suite: anesthesia, surgery, and nursing. Additionally, in the cardiac OR there is a subtribe, if you will, represented by the complex interaction between the perfusionist and the surgeon. So in this study the analysis focused on the surgical tribe in the surgical cockpit. Critical to our understanding of patient safety issues, the protocol for communication during CPB between the surgeon and the perfusionist has been established to some degree.

First, Dr Sundt, in your analysis of the cognitive workload of various participants in cardiac surgery and your use of the sterile cockpit analogy, you propose that we regard critical events as opposed to critical intervals as being more appropriate. My first question relates to the definition of "critical" and whether there is a measurable threshold for such definition, in view of the variability of what a given surgeon or anesthesiologist would consider a critical event. Is there a reliable threshold for what you would consider a critical event, given the variability of tasks and perceptions?

Dr Sundt. It's hard to know the answer to that. We did it on the basis of the potential impact of a problem at the time of that event, if you will, a consensus agreement on the potential for harm. If we screwed that step up, how bad would it be? We also looked at how often there were miscommunications surrounding a given event. I agree with you that this is an issue with all of this kind of science, or social science. It is hard to measure these things. Now if we accept that there is no standardized or completely objective way to determine critical events, the implication is that there are multiple definitions. I can personally accept the notion that from institution to institution the definitions of those critical events may vary somewhat. But there is a limit, too. If the definitions vary from OR to OR, from surgeon to surgeon, from anesthesiologist to anesthesiologist, then we basically have the very state that we have right now, which may work if one always has the exact same team and everyone knows the definitions. But I don't think that is going to be an adequate answer in the long run. I think that in the end we need to come to some agreements, at least within our own work groups. It is an interactive process. In our institution, we work on it, modify, and I think that we can come to some kind of common ground in that way.

Dr Fann. Most surgeons and perfusionists have developed an effective working relationship and means of communication with respect to terminology, phraseology, call-back, nuances, and so on. Given the number of surgeons and perfusionists in the study, did you find in your subanalysis that there tended to a breakdown in communication with particular combinations of surgeons and perfusionists? If so, the communication protocol you developed is actually a means of remediation for those individuals.

Dr Sundt. The surgeons and perfusionists involved in this study were a subset of individuals who were open to doing this, so if anything they are probably the people who were going to be most pliable and for whom the communication was already most reliable. I mentioned the issue about ACT. If I can digress for a moment, we changed a heparin administration protocol not too long ago, and the next week I got complaints, "Oh, with this change we almost went on CPB several times without giving heparin." The question was, how could this possibly happen? I asked the perfusionists how they could institute CPB without an adequate ACT. The response I got from the perfusionist was, "Well, Dr So-and-So goes on CPB without asking what the ACT is." I have to admit I asked the perfusionist, "What do you mean Dr So-and-so goes on CPB? Aren't you the perfusionist? Aren't you the one who turns on the machine?', Of course, the perfusionists are not personally at fault-this is the culture in our institution, and I'll bet that we are not unique. So I think the answer to your question is that there are definitely very strong differences among the surgeons regarding how open they are to communication and how open the communication is in their ORs. And, yes, I think that addressing this issue is very much the intent of the work, to try and smooth that out. Because poor communication in one OR spills over and affects what happens in another OR.

Dr Fann. We know that when things get tough during surgery such technically difficult components of the case are not always known to the rest of the team because of inability of anyone but the surgeon in many cases to visualize the situation and because of the unpredictable nature of some of these events. In these situations, the surgeon would often communicate the critical nature of the event to the rest of the team to minimize the amount of background noise and distraction. So, because of the unpredictable nature of the critical events, especially during a long operation, and the nature of multiple cockpits, based on your observations, have you identified effective or not so effective methods that surgeons use to communicate such critical events?

Dr Sundt. I think that the important principle there is that the surgeon needs to communicate the criticality of the situation in a way that actively engages everyone in the room, rather than disengaging everyone in the room. Maybe this is more in the realm of opinion than fact, but if we throw an instrument and it makes everybody afraid to speak up, then we actually have not accomplished our goal. Our goal really was to get everybody's head in the game. Instead, what we just got by throwing an instrument was for everybody to check out of the room. There are better ways to command attention. I think that we have all seen role models for this in our training, people who commanded the attention of those around them with their quiet manner. I think that is probably the most effective way to get everybody actively engaged.

Dr Fann. Finally, what are the implications of your findings for crew resource management training and crisis management training in current patient simulation training? That is, crew resource management has been effective in anesthesia simulation, but simulation 
of surgeon-perfusionist interactions appears to be necessary as well. Please comment on this issue.

Dr Sundt. We are beginning to explore the use of simulation in that axis. Harold Burkhart will actually discuss some of that tomorrow, I think. We have a perfusion simulator in our simulation center and are trying to get surgeons and perfusionists together there to look at what those interactions are and how they occur.

Dr Kent Jones (Salt Lake City, Utah). I'll be brief; I just have one question. In you showed improvement in all categories except that designated as miscues.

Dr Sundt. Miscues.
Dr Jones. Yes. Would you not have expected that area to improve with all the other categories that did show improvement?

Dr Sundt. The single category that did not show an improvement was miscues. The difference between the frequencies was not statistically significant, for what statistical significance is worth. I would expect that certainly not to have been worse, so I can make the excuse that it was not a statistically significant difference. The incidence of those miscues was already pretty low, perhaps because there was a Hawthorne effect in this. Everybody knew what we were doing in the room, and so they were paying more attention, but it turned out that the difference was not statistically significant. 